\title{
Diseño basado en diagnóstico de fallos y sistemas híbridos aplicado en un equipo de desfibrilación ventricular
}

\author{
Design based on fault diagnosis and hybrid systems applied \\ to a ventricular defibrillator device
}

\author{
Alberto Prieto Moreno ${ }^{1}$ \\ Orestes Llanes-Santiago ${ }^{1}$ \\ Alejandro Milanés-Cruz ${ }^{2}$ \\ José Folgueras-Méndez ${ }^{2}$ \\ Emilio García-Moreno ${ }^{3}$ \\ Francisco Morant-Anglada ${ }^{3}$
}

Recibido 7 de noviembre de 2011, aceptado 10 de abril de 2012

Received: November 7, $2011 \quad$ Accepted: April 10, 2012

\begin{abstract}
RESUMEN
En este artículo se presenta una propuesta de procedimiento que incorpora el diagnóstico de fallos desde la fase de diseño de un equipo de desfibrilación ventricular. Lo anterior permite resolver un grupo de limitaciones que están presentes actualmente en el diseño de sistemas electrónicos.

El procedimiento propuesto utiliza el concepto de diseño basado en diagnóstico, la técnica de composición de autómatas híbridos para el modelado y diagnóstico basado en el conocimiento de los expertos. Finalmente se diseña el sistema con el diagnosticador ya incorporado. El procedimiento utilizado puede ser extendido a otros tipos de sistemas.
\end{abstract}

Palabras clave: Sistema de diagnóstico de fallos (SDF), diseño basado en diagnóstico, autómatas híbridos, autómatas cronometrados, composición de autómatas, árboles de decisión.

\section{ABSTRACT}

This article presents a proposal of procedure to incorporate the fault diagnosis from the design phase of ventricular defibrillation equipment. This solves a set of constraints that are currently present in the design of electronic systems.

The proposed procedure uses the concept of diagnosis-based design, the technique of composition of hybrid automata modeling and diagnosis based on expert knowledge. Finally the system is designed with the fault diagnostic system incorporated. The procedure can be extended to other types of systems.

Keywords: Fault diagnosis system, diagnosis-based design, hybrid automata, timed automata, automata composition, decision tree.

\section{INTRODUCCIÓN}

El diagnóstico de fallos, que es el proceso de detección y localización de un fallo, es una temática en la que se ha trabajado intensamente en los últimos 30 años y que cuenta hoy con un número importante de resultados teóricos y prácticos. Estos resultados se han expuesto y publicado en numerosos congresos y revistas, lo que demuestra que esta área ha recibido considerable atención, tanto del mundo industrial como del académico, por el gran impacto económico y de seguridad que trae consigo.

1 Departamento de Automática y Computación. Facultad de Ingeniería Eléctrica. I.S.P.J.A.E. Calle 114, No 11901 e/ 119 y 127. CUJAE, Marianao, Ciudad de La Habana, Cuba. E-mail: albprieto@electrica.cujae.edu.cu; orestes@electrica.cujae.edu.cu

2 Instituto Central de Investigación Digital (ICID). Calle 202, No. 1704 entre 17 y 19. Siboney, Playa, La Habana. E-mail: amilanes@icid.cu; jfolgueras@icid.cu

3 Departamento de Ingeniería de Sistemas y Automática, Universidad Politécnica de Valencia. C. Ver, 14, Apdo 22012-46071. Valencia, España. E-mail: egarciam@isa.upv.es; fmorant@isa.upv.es 
Son varios los enfoques que se presentan en la literatura para el diagnóstico de fallos [1]. Uno de los más difundidos es la aplicación de las técnicas basadas en redundancia analítica a partir de la utilización de modelos cuantitativos [2,3], las cuales pueden agruparse en enfoques tales como el de espacios de paridad [4], observadores de estado [5] y estimación e identificación de parámetros [6]. Otros enfoques son la aplicación de técnicas de inteligencia artificial para capturar el conocimiento experto y la utilización de técnicas estadísticas multivariada y de reconocimiento de patrones, para el análisis y caracterización de los datos históricos que reflejan el comportamiento del sistema bajo diferentes estados de funcionamiento. Aunque estos enfoques se presentan de forma separada en los trabajos de revisión [7-9], es muy común encontrarlos como aplicaciones complementarias [10-12].

El aumento de la productividad y la obtención de nuevas prestaciones en los equipos tecnológicos ha sido posible gracias a la utilización de técnicas de diseño de sistemas digitales cada vez más sofisticadas que llevan implícito la utilización de un desarrollo electrónico cada vez más complejo.

Este crecimiento en la complejidad de los equipos provoca que la probabilidad de ocurrencia de fallos sea cada vez mayor y la posibilidad de diagnosticarlos sea cada vez más difícil. A esto hay que adicionar las restricciones que se le imponen al personal encargado del diseño por cuestiones de costo y prestaciones del equipo. Entre algunas de estas restricciones se pueden citar:

- Evitar la utilización de redundancia física debido a la implicación directa en el costo que esta trae consigo.

- Evitar hacer pruebas de desempeño de los procedimientos de diagnóstico sobre los sistemas físicos por problemas de costo, lo que genera la necesidad de contar con modelos dinámicos para realizar dichas pruebas.

- Existen procesos críticos en el tiempo que no admiten la redundancia analítica como estrategia de diagnóstico.

- Existe poca información disponible del estado del sistema para realizar las tareas de diagnóstico.

- La sensibilidad de los sistemas de diagnóstico de fallos (SDF) ante los errores de modelado y las perturbaciones externas es un aspecto muy importante a tener en cuenta.
Actualmente la mayoría de los sistemas electrónicos son diseñados con un sistema de protecciones para evitar que el fallo de algún componente pueda dañar a otra parte del sistema y/o a los usuarios del mismo. Sin embargo, en un grupo considerable de casos las protecciones no son usadas convenientemente para poder localizar el causante del fallo, lo cual provoca demoras innecesarias en los procesos de reparación.

En este trabajo se presenta una propuesta para incorporar desde la fase de diseño de equipos electrónicos la concepción de un sistema de diagnóstico de fallos que logra resolver algunas de las restricciones anteriores, dotando de nuevas prestaciones a los sistemas diseñados y facilitando los procesos de mantenimiento y reparación. Lo anterior implica mejorar la confiabilidad operacional del equipo.

La estructura de este trabajo es la siguiente: en la sección 2 se presenta el procedimiento general a seguir. La sección 3 muestra las principales características y el diagrama en bloques general de un equipo desfibrilador. En la sección 4 se presenta la aplicación del procedimiento propuesto al bloque de carga del capacitor de un desfibrilador ventricular. Esto incluye: la definición de los fallos a detectar, la determinación de las variables necesarias para este propósito, el modelado matemático del sistema a diagnosticar y la selección e implementación del método de diagnóstico a partir del análisis de las diferentes alternativas y la validación del diagnosticador. Finalmente se presentan las conclusiones.

\section{PROCEDIMIENTO PARA INCORPORAR UN SDF DESDE LA FASE DE DISEÑO EN SISTEMAS ELECTRÓNICOS}

En la mayor parte de la bibliografía existente en la temática de diagnóstico de fallos, las técnicas desarrolladas parten de un sistema ya realizado. A partir de las condiciones de observabilidad del mismo, se busca la separabilidad en el diagnóstico de cada uno de los elementos que integran el conjunto de fallos establecido para el sistema.

Como se sabe, la observabilidad de un sistema es consecuencia directa del número de sensores que se utilicen. En el entorno industrial, de manera general, no suele ser un aspecto crítico la modificación de 
los sistemas para incorporar nuevos sensores cuando se decida la adquisición y seguimiento de nuevas variables con fines de diagnóstico. Sin embargo, en el caso de los sistemas electrónicos esto sí es un problema crítico. Una vez construida la placa con el circuito electrónico, la incorporación de nuevos sensores implica desechar la placa original y la pérdida de la mayoría de las componentes que estaban en ella. Es por esta razón que para este tipo de sistemas se propone incorporar el concepto de diseño basado en criterios de diagnóstico.

Sea $S$ la descripción del funcionamiento de un sistema deseado y $Q_{f}$ el conjunto de fallos que se desea detectar en el funcionamiento del mismo.

El diseño basado en criterios de diagnóstico establece que desde la fase de diseño del sistema $S$ se debe indicar el conjunto de fallos $Q_{f}$ que se desea diagnosticar con el objetivo de garantizar las condiciones de observabilidad necesarias para el diagnóstico a partir de un análisis de factibilidad. Este conjunto de fallos se obtiene a partir del conocimiento de los expertos y/o de la aplicación de metodologías inductivas y deductivas que permitan conocer los modos de fallos con mayor probabilidad de ocurrencia o los modos de fallos estadísticamente con mayor riesgo de no funcionalidad.

Tomando en consideración lo anterior se presentan los dos primeros pasos a realizar, una vez que ya esté la concepción de diseño del sistema:

\section{Definir los fallos a detectar}

Desde la fase de diseño del sistema se deben definir los fallos fundamentales a diagnosticar que afectarían el funcionamiento deseado del sistema. Como la mayoría de los diseños presentan elementos novedosos, es primordial prestar atención a los problemas de fallos que se van presentando en los procesos de montaje y puesta a punto ya que ellos son indicadores de las posibles insuficiencias presentes en el diseño. Conjuntamente a esto es imprescindible consultar la opinión de los expertos y además considerar criterios prácticos de mantenimiento, reparación y costo/beneficio.

\section{Seleccionar las variables a medir}

Una vez definidos cuáles son los fallos que se desea diagnosticar, se deben indicar las variables necesarias a medir, o sea, las variables en cuyo comportamiento se basará la estrategia de diagnóstico para determinar el estado del sistema. Este es un aspecto clave ya que determina la cantidad de sensores que son necesarios utilizar para el seguimiento del estado del sistema, lo que determina las modificaciones que se deben realizar sobre el esquema de funcionamiento básico del equipo. En este punto además, como una forma posible de disminuir costos a partir de reducir el número de sensores a utilizar, debe analizarse la posibilidad de obtener mediante redundancia analítica el valor de algunas de las variables seleccionadas para medir.

Una vez concluidos los dos pasos anteriores ya se tiene un diseño del sistema basado en criterios de diagnóstico.

En la mayoría de los casos es recomendable utilizar un modelo del dispositivo para evaluar, en una primera fase, el desempeño del sistema de diagnóstico mediante simulación sin tener necesidad de utilizar prototipos del sistema. Entonces, el siguiente paso en el diseño del sistema es:

\section{Obtener y validar el modelo del dispositivo}

Para la obtención del modelo se podrían utilizar diversas herramientas existentes; sin embargo, se recomienda utilizar los resultados recientes de la teoría de sistemas híbridos [13-15], ya que estos brindan la posibilidad de modelar sistemas dinámicos con variables de estado que toman valores continuos y discretos. También permite simplificar los niveles de complejidad en el modelado del funcionamiento real del dispositivo, lo que posibilita obtener el nivel de precisión que se desee. Además, brinda la posibilidad de incorporar de forma bien definida, dentro de un modelo general, las características de cada uno de los subsistemas por los que está compuesto el equipo.

Como es clásico en cualquier procedimiento que incluya trabajo con modelos, una vez que este es obtenido debe ser validado.

\section{Seleccionar el método de diagnóstico a utilizar y diseñar el diagnosticador}

Para seleccionar el método de diagnóstico a utilizar se debe realizar un análisis de las características del dispositivo a diagnosticar y los requerimientos de cada uno de los enfoques generales de diagnóstico. En la mayoría de los casos la demanda de capacidad 
computacional y de almacenamiento, así como el tiempo de cálculo requerido, son aspectos fundamentales a evaluar.

Para implementar estrategias de diagnóstico basadas en modelo se requiere disponer de un modelo que describa de manera satisfactoria el dispositivo a diagnosticar. Como es conocido, en general estos métodos consumen una cantidad de tiempo relativamente grande en su ejecución, aspecto que los limita en dispositivos con restricciones de tiempo en su funcionamiento, como es común en muchos sistemas electrónicos.

Para el caso de estrategias de diagnóstico basadas en la historia del proceso, se requiere de una gran cantidad de datos en los cuales se reflejen todos los fallos que se desea diagnosticar [16, 17]. Esta es una necesidad que suele satisfacerse sin problemas en procesos industriales, gracias a la utilización de los sistemas SCADA y que pudiera ser factible en algunos sistemas electrónicos. Sin embargo, esta característica de almacenamiento masivo de las variables que se adquieren y siguen para su análisis posterior, no es una característica en la mayoría de los sistemas electrónicos. Además, los métodos utilizados bajo este enfoque demandan una elevada capacidad de cómputo y tiempo para los cálculos.

Otras alternativas para el diagnóstico se pueden encontrar utilizando estrategias basadas en el conocimiento de expertos. Este enfoque ofrece un espectro amplio de métodos a utilizar donde varían los requerimientos demandados en cada uno de ellos $[18,19]$. Debe destacarse que incorporar conocimiento de expertos es, en sentido general, un aspecto muy ventajoso en tareas de diagnóstico.

Como puede apreciarse, en la selección del método de diagnóstico a utilizar juegan un papel fundamental el conocimiento disponible sobre el sistema que se diseña, las características del mismo y los regímenes de funcionamiento a los que está sometido.

Después de seleccionada la estrategia adecuada de diagnóstico, entonces se pasa al diseño del diagnosticador.

\section{Evaluar el desempeño del diagnosticador}

Con este fin se deben definir los índices de acierto en el diagnóstico, considerando la cantidad de falsas alarmas, los fallos no detectados y la rapidez del diagnóstico. Si las pruebas realizadas al diagnosticador no son satisfactorias debe regresarse al paso dos y revisarse nuevamente cada punto buscando un refinamiento de todo el análisis realizado. En caso de que las pruebas cumplan con los requerimientos fijados es posible pasar al último paso del procedimiento.

\section{Implementar el diagnosticador}

En la implementación en línea deben utilizarse los métodos más eficientes en cuanto a consumo de memoria y tiempo de ejecución, buscando no sobrecargar al sistema, ya que el diagnosticador es un aspecto más dentro de este pero no es su objetivo principal.

La Figura 1 muestra un diagrama resumen de los pasos involucrados en el proceso de diseño basado en diagnóstico.

El enfoque propuesto fue aplicado en el diseño de un equipo de desfibrilación ventricular y puede ser extendido al diseño de otros sistemas electrónicos $\mathrm{y}$ al diseño de sistemas industriales.

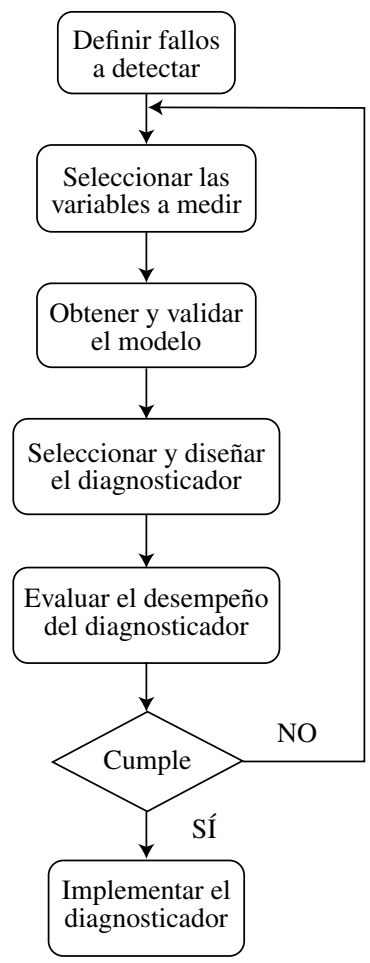

Figura 1. Diagrama de secuencias del diseño basado en criterios de diagnóstico. 


\section{ALGUNAS CARACTERÍSTICAS Y DIAGRAMA EN BLOQUES GENERAL DE UN EQUIPO DESFIBRILADOR VENTRICULAR}

El paro cardíaco continúa siendo un azote de la sociedad moderna que afecta a un elevado número de personas anualmente, generalmente debido a la fibrilación ventricular o patologías asociadas. Según algunos estudios, alrededor de un $40 \%$ de estas personas se encuentran en fibrilación ventricular, la cual tiene en la desfibrilación eléctrica un tratamiento rápido y efectivo, capaz de terminarla.

En el protocolo de la American Heart Association (AHA) para desfibrilación se recomienda la aplicación de una energía progresiva de 200 J-300 J-360 J cuando se emplea la forma de onda monofásica. Sin embargo, cuando la forma de onda empleada es la bifásica, basta con emplear un protocolo de $100 \mathrm{~J}-130 \mathrm{~J}-180 \mathrm{~J}$ para lograr resultados más eficaces que los obtenidos con el protocolo antiguo [20].

Para ejemplificar la propuesta que se hizo en la sección anterior se utilizó el Desfibrilador-Monitor CARDIODEF2 [21], diseñado y desarrollado en el Instituto Central de Investigación Digital (ICID), en La Habana, Cuba.

El esquema de bloques funcionales de este equipo se muestra en la Figura 2. Como puede apreciarse en [22], este es un diagrama funcional general para la mayoría de los equipos desfibriladores.

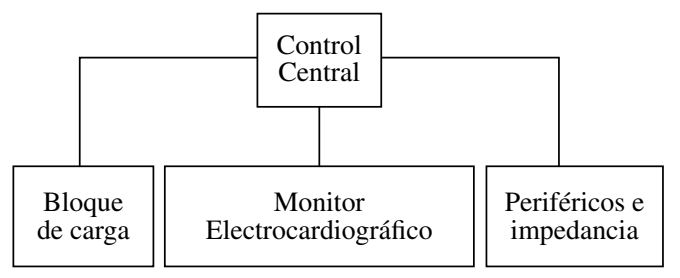

Figura 2. Bloques funcionales del CARDIODEF2.

El bloque funcional en el que hay que poner un énfasis especial, con relación a la seguridad, es el de carga del capacitor. Es aquí donde se almacena la energía necesaria para la descarga eléctrica a aplicar, por lo que es muy importante tener un control de la misma para que se encuentre en correspondencia con la seleccionada, evitando daños colaterales debido a la aplicación de una energía excesiva y a la vez lograr una resucitación efectiva. Esto explica la selección de este bloque como ejemplo para la aplicación de la propuesta de procedimiento.

\section{DISEÑO DEL BLOQUE DE CARGA DEL CAPACITOR}

Se presenta a continuación la aplicación del enfoque propuesto en el bloque de carga del equipo desfibrilador.

\section{Definición de los fallos a diagnosticar y las variables a medir}

El bloque de carga del desfibrilador CARDIODEF2 está constituido, en lo fundamental, por un convertidor de corriente continua tipo elevador con aislamiento de la entrada ("flyback"), un oscilador que genera un tren de pulsos con ancho modulado, los circuitos electrónicos de medición y trasmisión y un microcontrolador que tiene la misión de controlar todo el proceso de la carga del capacitor [21].

En la fase de diseño del equipo desfibrilador se utilizó el concepto de diseño basado en diagnóstico, el cual implica establecer desde esta fase los fallos que debían detectarse para garantizar seguridad y facilitar las labores de producción, mantenimiento y reparación. En este sentido, después del diseño preliminar se estableció que el diagnóstico indicaría fallos a nivel de bloque funcional dentro del bloque de carga, con lo cual se obtendría la identificación de fallos en los siguientes bloques:

- Transformador-Capacitor

- Oscilador

- Sistema de Medición

Esta propuesta de trabajar a nivel de bloques funcionales está determinada por dos factores: el procedimiento de reparación de los equipos, y lograr mejores resultados desde el punto de vista costo-beneficio.

Por lo general, con el objetivo de disminuir el tiempo que se encuentra fuera de servicio un equipo, es una práctica frecuente la sustitución de bloques funcionales en lugar de la sustitución de elementos dañados. Para el sistema bajo estudio se consideró utilizar esta práctica, decisión que se considera acertada si se tiene en cuenta que para que el equipo sea capaz de identificar el componente dañado 
dentro de un bloque funcional implica tener que medir muchas más variables, con su correspondiente aumento del costo de producción, y desarrollar algoritmos de diagnóstico de mayor complejidad.

Por lo tanto, se decidió que los diferentes fallos que pueden ocurrir dentro de los bloques funcionales serían diagnosticados como un fallo del bloque completo y no de algún componente específico. Esto hace que el diagnosticador que se implemente en el equipo no tenga que ofrecer una identificación del componente específico que falló, simplificando la complejidad del mismo y cumpliéndose a su vez con el procedimiento de mantenimiento establecido para el equipo.

\section{Análisis del bloque transformador-capacitor}

El proceso de carga del capacitor queda descrito por tres variables de estado: la corriente en el primario del transformador $i_{p}$, la corriente en el secundario $i_{s}$ y la tensión en el capacitor $V_{c}$. Los diseñadores definieron un perfil (o curva) del comportamiento del proceso de carga normal, el cual fue tabulado indicando el rango de valores $V_{n c} \pm \theta$ en los que debe encontrarse la tensión en el capacitor a medida que transcurre este proceso. $V_{n c}$ es el valor nominal que debe tener la tensión en el capacitor $V_{c}$, en un instante del proceso dado y $\theta$ está en relación con los niveles de ruido existentes en el proceso de transferencia de energía hacia el capacitor, buscando la forma de hacer al sistema lo más inmune posible al ruido. Este perfil tabulado no es más que un indicativo de la velocidad que debe tener el proceso de carga del capacitor.

Un fallo en alguno de los elementos que forman este sistema siempre se reflejaría en la dinámica que describe el comportamiento del proceso de carga del capacitor. Se utiliza la comparación, en instantes específicos del proceso de carga, entre el valor real de $V_{C} y$ los valores tabulados para la generación del residuo. Alguna discrepancia entre el comportamiento esperado y el real, o lo que es lo mismo, una desviación no permitida con relación al perfil definido, es indicativo de que uno de los elementos del bloque funcional está dañado. Las condiciones $V_{C}<V_{n c}-\theta$ ó $V_{C}>V_{n c}+\theta$ analizan la velocidad de carga del capacitor. Una medición que indique que la tensión está fuera de rango sería reflejo de la existencia de un fallo.

\section{Análisis del bloque oscilador}

El tiempo que van a estar activos los diferentes estados involucrados en la carga del capacitor $t_{o n}$, tiempo de almacenamiento de energía en el primario del transformador $\mathrm{y} t$, tiempo de almacenamiento de energía en el capacitor, dependen directamente de la duración de los estados: nivel alto y nivel bajo de la salida del oscilador. La violación de las condiciones definidas por los diseñadores $t_{o n} \leq 0.75 T$ y $t_{p} \leq T$ donde $T$ es el período del oscilador, reflejaría la existencia de mal funcionamiento en este bloque.

Utilizar un contador de tiempo que verifique la duración de dichos estados sería engorroso y además requeriría de una mayor cantidad de componentes en el sistema. Es por eso que se decidió utilizar la variable de estado $i_{p}$ para la generación de residuos indicadores del estado del bloque oscilador. Si esta variable sobrepasa ciertos límites significa que la duración de los pulsos no es la establecida. Es necesario aclarar que la variable $i_{p}$ también es afectada por el valor de la fuente $E$ y de la inductancia en el primario $L_{p}$. En caso de una variación de $L_{p}$ esta se reflejaría en la velocidad de carga del capacitor y por lo tanto saldría como un fallo en el bloque que anteriormente analizamos.

Problemas en la fuente $E$ son detectados en otro de los subsistemas del desfibrilador que garantizan el funcionamiento del equipo. Todo lo anterior asegura que diagnosticar fallos del oscilador utilizando la medición de $i_{p}$ sea robusto a la variación de los parámetros del sistema.

\section{Análisis del bloque sistema de medición}

A diferencia de los casos anteriores, los residuos en este bloque no se generan utilizando los modelos que describen el comportamiento correcto de las variables de estado, sino se generan buscando valores absurdos o extremos de las variables de estado, los cuales serían resultados de fallos en este bloque. Por ejemplo, la condición $V_{c}=V_{n c}+\theta$ sería un valor absurdo de la medición ya que no es posible que el capacitor tenga almacenada más energía de la que se le ha transferido hasta ese momento. Como la variable $V_{c}$ es muy importante, se decidió crear una redundancia física en la medición, la cual posibilita detectar otra condición de fallo de este bloque. Esta condición se alcanza cuando la tensión en el capacitor se iguala a la máxima permitida para el 
sistema $V_{C}=V_{M P}$, indicando que se ha sobrepasado el valor de tensión deseado.

Adicionalmente, hay que señalar que la carga del capacitor debe realizarse en el tiempo establecido por los fabricantes como la máxima duración permitida para este proceso. No cumplir con la condición $t_{c}<5 \mathrm{~s}$, donde $t_{c}$ representa el tiempo de carga transcurrido, será indicativo de la ocurrencia de un fallo en el sistema.

Definición 1. Se define $F$ como el conjunto de los fallos en el bloque de carga que estará formado por $F=\left\{F_{1}, F_{2}, F_{3}\right\}$ donde:

\section{$F_{1}$, Falloen el bloque Transf-Capacitor \\ $F_{2}$, Falloen el bloque Oscilador \\ $F_{3}$, Falloen el bloque Sistema de Medición}

La definición anterior puede verse como que $F_{1}$ será el diagnóstico que indicará el equipo cuando ocurra algún fallo en el bloque Transformador-Capacitor como pudieran ser: roturas del capacitor, apertura o cortocircuitos en los devanados de transformador. Esto es así porque se decidió que la identificación del componente dañado dentro del bloque se realizaría en el proceso de reparación, a fin de no sobrecargar el funcionamiento del equipo con un proceso de diagnóstico más complejo. Lo mismo se aplica para el diagnóstico de los respectivos fallos que pueden ocurrir en los dos bloques funcionales restantes que componen el sistema bajo análisis.

De acuerdo con la experiencia de los expertos, la probabilidad de ocurrencia de fallos múltiples es muy baja. Es por esto que, con el objetivo de simplificar el proceso de diagnóstico, se estableció que:

Definición 2. Se define $O_{c}$ como el conjunto de ocurrencias de los fallos $F_{i}$ de manera tal que $O_{c_{F_{1}}} \cap O_{c_{F_{2}}} \cap O_{c_{3_{1}}}=\varnothing$.

Lo anterior significa que el diagnosticador trabajará con el supuesto de que solo ocurre un fallo en un instante de tiempo. Aunque en la práctica no siempre se cumpla este supuesto y puedan presentarse fallos múltiples, esta situación no traería alguna implicación no prevista. Una vez que el equipo diagnostica un fallo en alguno de los bloques analizados, es retirado del servicio. En el proceso de reparación será sustituido el bloque funcional causante del fallo y cuando sea probado nuevamente antes de incorporarse al servicio, entonces se diagnosticará el otro fallo que había ocurrido. Este proceso permite que todos los fallos sean eliminados sin la necesidad de complejizar el algoritmo del diagnosticador.

A partir del análisis de cada bloque funcional y de conjunto con el criterio de los expertos, se determinó que los parámetros importantes para darle seguimiento en el equipo desfibrilador debían ser:

- Velocidad de carga del capacitor

- Corriente en el devanado primario del transformador

- Tensión máxima permitida

- Tiempo máximo de carga permitido

\section{Modelado del sistema a diagnosticar y validación del modelo obtenido}

Una vez definidos los fallos a detectar y las variables a medir para su utilización en el diseño final, corresponde entonces obtener el modelo del sistema para comprobar mediante simulaciones los resultados esperados.

Típicamente los sistemas de ingeniería poseen una estructura jerárquica, caracterizada por variables dinámicas continuas en el nivel más bajo y mecanismos de toma de decisiones lógicas en el nivel más alto, por lo que coexisten variables de estados continuas y estados discretos. Tradicionalmente, la naturaleza híbrida de estos sistemas es suprimida convirtiéndolos en entidades puramente discretas o continuas.

Motivados por representar el problema lo más cercano a la realidad surge en la década de 1990-1999 el concepto de "sistemas híbridos" como colecciones de sistemas dinámicos interactuantes en los que evolucionan variables de estado continuas sujetas a cambios discretos.

El reciente interés por los sistemas híbridos ha sido estimulado por varios factores. Para los teóricos del control, es un reto intelectual extender conceptos fundamentales tales como estabilidad y controlabilidad para los sistemas híbridos. Los teóricos de ciencia de la computación, por otra 
parte, se interesan en extender los métodos formales para tratar con sistemas de estados discretos a sistemas dinámicos con estados continuos [13]. Para ambas ramas de la ciencia, el conjunto principal de aplicaciones es el control de sistemas físicos dinámicos usando sistemas digitales.

La teoría de sistemas híbridos ha desarrollado muchos modelos y técnicas de análisis y síntesis, encontrando aplicación en numerosas áreas entre las que se encuentran la aeronáutica [23], el control de automóviles [24], sistemas de transportación [25] y robótica móvil [26].

Dos grandes ventajas que tiene representar los sistemas mediante modelos híbridos son:

- Reducción del grado de complejidad en la representación del comportamiento dinámico de los mismos, gracias a la partición que puede hacerse del modelo global en diferentes niveles de menor complejidad.

- Se evita el trabajo con un conjunto de ecuaciones no lineales, debido a la posibilidad de escoger conjuntos de ecuaciones más simples (lineales) y conmutar entre modelos más simples.

Son las ventajas anteriores las que motivan escoger la representación mediante modelos híbridos para modelar los sistemas que se están diseñando.

El autómata híbrido se ha convertido en un modelo estándar para sistemas híbridos y es en esencia un autómata de estados finitos aumentado con un vector de variables de estado continuas y una ecuación de estados continuos para cada estado discreto [13].

No es un objetivo de este trabajo profundizar en la teoría de los sistemas híbridos, por lo que solo se presentan los conceptos fundamentales relacionados con esta técnica de modelado que fueron utilizadas para el modelado del bloque de carga del capacitor.

Un autómata híbrido se define como en [14]:

Definición 3. Un autómata híbrido se describe por $H=\left(Q, X, \Sigma, I, I_{n v}, E, f\right)$ donde $Q$ es un conjunto finito de estados discretos o modos del sistema, $X \subseteq \mathfrak{R}^{n}$ es el espacio de estados continuos, $\Sigma$ es un conjunto de identificadores de transición o eventos,
$I \subseteq Q \times X$ es el conjunto de condiciones iniciales, $I_{n v}: Q \rightarrow 2 X$ es el invariante asociado con cada modo $q, E \subset Q \times X \times \Sigma \times Q \times X$ es el conjunto de transiciones discretas, y $f: Q \times X \rightarrow X$ es la condición de flujo para todos los modos.

Para los autómatas híbridos que pueden representar sistemas cuyos comportamientos presenten restricciones de tiempo, se aumenta el vector de estado con variables que representan relojes, constituyendo lo que se conoce como autómata cronometrado.

Un autómata cronometrado se define como en [15]:

Definición 4. Un autómata cronometrado $C$ está formado por un conjunto ordenado $\left\langle L, L_{0}, \Sigma, X, I, E\right\rangle$, donde:

- $L$ es un conjunto finito de localizaciones,

- $L_{0} \subseteq L$ es un conjunto de localizaciones iniciales,

- $\Sigma$ es un conjunto finito de identificadores,

- $X$ es un conjunto finito de relojes,

- $\quad I$ es un mapeo que identifica cada localización $l$ con alguna restricción de tiempo en $\Phi(X)$,

- $E \subseteq L \times \Phi(X) \times L$ es un conjunto de cambios. Un cambio $\left\langle l, a, \phi, \lambda, l^{\prime}\right\rangle$ representa una arista desde la localización $l$ hacia la localización $l^{\prime}$ con identificador $a$. $\varphi$ es una restricción de tiempo sobre $X$ que especifica el cambio es habilitado, y el conjunto $\lambda \subseteq X$ da los relojes que deben ser reiniciados con ese cambio.

Cuando un sistema es modelado por diferentes autómatas que representan subsistemas que interactúan entre sí, el modelo del sistema global se obtiene a partir del producto de los autómatas que modelan los subsistemas, formando una composición paralela de autómatas. Una definición formal de este concepto puede encontrarse en [14].

A los efectos de obtener el modelo del dispositivo para su utilización en las diferentes fases del proceso de diagnóstico, el bloque de carga del desfibrilador se representó por tres subsistemas: convertidor "flyback", oscilador y reloj, los cuales se modelaron utilizando autómatas híbridos y autómatas cronometrados, con los que se logra una representación mucho más general para los sistemas dinámicos a partir de determinado nivel de complejidad. 
Es necesario destacar que no se presenta un modelo para el subsistema de medición a pesar de ser uno de los bloque funcionales, porque el diagnóstico del bloque Sistema de Medición se obtiene a partir de encontrar valores no lógicos o desviaciones en las mediciones realizadas en los subsistemas modelados.

Modelo híbrido del convertidor de corriente directa del tipo elevador con entrada aislada (flyback).

El esquema simplificado de un transformador de corriente directa del tipo elevador con entrada aislada se presenta a continuación en la Figura 3.

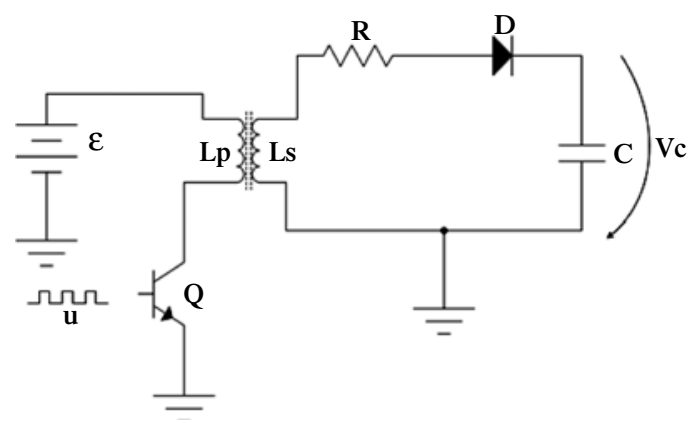

Figura 3. Convertidor flyback.

Este convertidor basa su funcionamiento en la conmutación del transistor $Q$ de corte $(u=0)$ a saturación $(u=1)$, lo cual identifica de manera inicial dos estados discretos bien definidos. En cada uno de esos estados discretos existe una dinámica continua que se formuló en base a la corriente en el primario del transformador $\left(i_{p}\right)$, la corriente en el secundario del transformador $\left(i_{s}\right)$ y la tensión en el capacitor $\left(V_{C}\right)$ [27], obteniéndose el modelo híbrido del convertidor flyback, el cual se representa en la Figura 4.

El estado $q_{0}$ describe la dinámica del proceso de almacenamiento de energía en el primario del transformador y el estado $q_{1}$ describe la dinámica del proceso de transferencia de energía desde el secundario del transformador hacia el capacitor. En este modelo se incluye un estado de fallo $q_{F}$ que se alcanza cuando el valor de tensión medido está fuera de los límites de tolerancia de desviación definidos por el fabricante, que es uno de los fallos que interesa detectar.

Desde el modo $q_{0}$ salen transiciones hacia los modos $q_{2}$ y $q_{F}$. La primera ocurre cuando se ha alcanzado la condición de tensión deseada en el capacitor, identificada por la condición $V_{C}=V_{c d}$. La otra transición ocurre cuando se ha detectado un fallo en el bloque funcional Transformador-Capacitor.

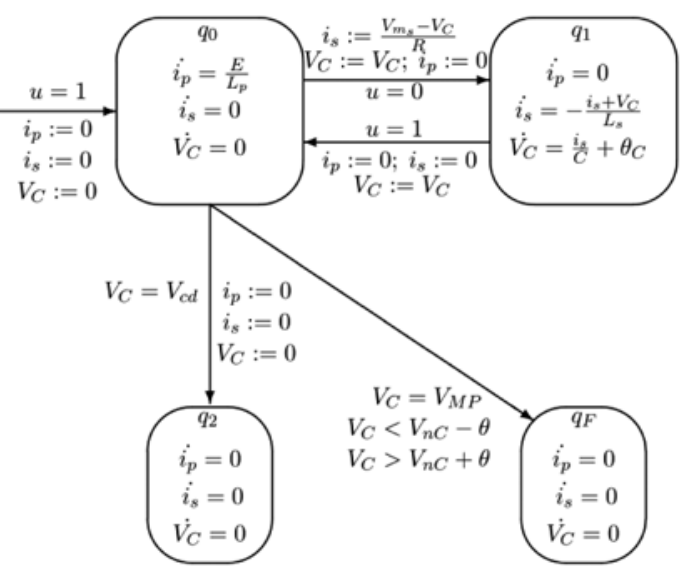

Figura 4. Modelo híbrido del bloque TransformadorCapacitor.

Autómata cronometrado del reloj

El reloj tiene la función de indicar el tiempo que lleva cargándose el capacitor, lo que permite conocer la duración de este proceso. El autómata cronometrado que modela el sistema de control de la duración del proceso se muestra en la Figura 5.

En este autómata, el reloj $t_{c}$ indica el tiempo transcurrido mientras el capacitor estuvo cargándose. Cuando se detecta que el capacitor ha alcanzado la tensión deseada, ocurre una transición desde el modo $q_{0}$ hacia el modo $q_{1}$, en donde se detiene el conteo del tiempo. La transición hacia el modo $q_{F}$ ocurre cuando el reloj $t_{c}$ no cumple con la condición invariante para el estado $q_{0}\left(t_{c}<t_{\text {lim }}\right)$. El tiempo $t_{\text {lim }}$ es el definido por el fabricante como el tiempo máximo que debe demorar el proceso de carga normal. Para

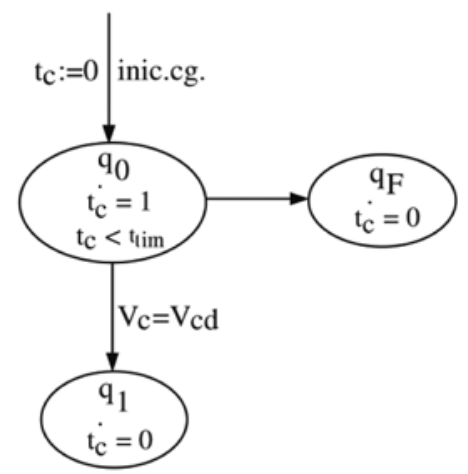

Figura 5. Autómata cronometrado del reloj. 
este sistema se establece que el proceso no debe durar más de 5 segundos, o sea, $t \mathrm{lim}=5 \mathrm{~s}$.

El modo $q_{F}$ no indica un fallo del reloj sino en el proceso de carga del capacitor.

\section{Autómata cronometrado del oscilador}

El oscilador es el dispositivo encargado de generar un tren de pulsos con ancho modulado para controlar la carga del capacitor. El autómata cronometrado representado en la Figura 6 modela el comportamiento del oscilador. Las transiciones hacia el estado $q_{F}$ representan los fallos en este subsistema. O sea, duración de los niveles de los pulsos fuera de los tiempos establecidos.

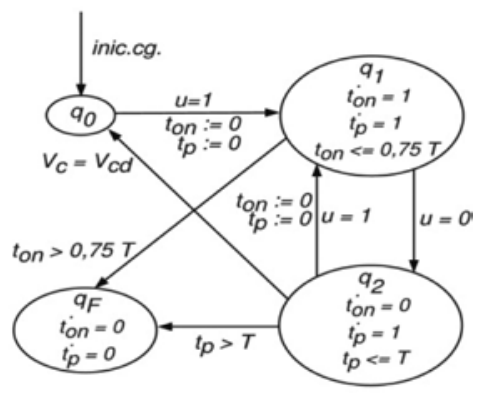

Figura 6. Autómata cronometrado del oscilador.

\section{Autómata paralelo}

Se construyó el autómata paralelo que modela el bloque de carga del capacitor a partir de los tres autómatas anteriores. El autómata resultante se muestra en la Figura 7.

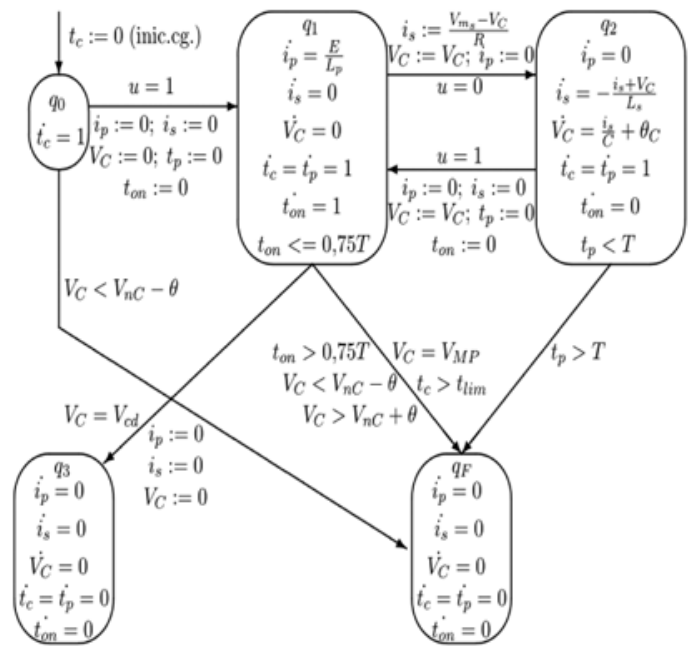

Figura 7. Modelo híbrido del proceso de carga del capacitor.
Este modelo se obtiene incorporándole al modelo híbrido del transformador-capacitor los relojes de los autómatas cronometrados del reloj y del oscilador, y combinando las diferentes restricciones que presentan.

Para la validación del modelo híbrido del proceso de carga del capacitor se hará un análisis comparativo entre las mediciones realizadas en el proceso real y el resultado de la simulación del modelo. El modelo se simuló utilizando el paquete Stateflow Toolbox de MatLab 7.0. [28] y los siguientes datos ofrecidos por los fabricantes:

$E=12 \mathrm{~V}-$ fuente de tensión

$L_{p}=3.2 \cdot 10^{-6} \mathrm{H}$-inductancia del enrollado primario

$L_{s}=98 \cdot 10^{-3} \mathrm{H}$-inductancia del enrollado secundario

$C=105 \cdot 10^{-6} \mathrm{~F}-$ capacidad del capacitor

$R=146.6 \Omega-$ resistencia interna del enrollado secundario

$r c=200-$ relación de conversión del transformador 4 vueltas en el enrollado primario y 800 en el enrollado secundario.

La Figura 8 muestra una parte de la evolución de las tres variables de estados del modelo híbrido mostrado en la Figura 7 que corrobora la transferencia entre los estados. Es fácil apreciar que en el estado $q_{1}$ aumenta la $i_{p}$ mientras $i_{s}=0$ y $V_{C}=c t e$. Luego en el estado $q_{2}$ la $i_{p}=0$ mientras que $i_{s}$ y $V_{C}$ varían con la dinámica descrita en el modelo.

\section{Análisis de los resultados de las mediciones}

Se realizaron tres corridas del proceso real buscando corroborar la repetitividad del mismo. Las curvas que se obtuvieron a partir de las mediciones realizadas, mostradas en la Figura 9, indican que la repetitividad del proceso es adecuada (hay una curva que coincide casi con otra, por lo que no se aprecia en la figura mostrada). Para facilitar todo el análisis se obtuvo la curva promedio de las mediciones $\mathrm{y}$, con respecto a esta, se calculó la función de autocorrelación, con la que se determinó que la cantidad de información que se tiene es suficiente y permite hacer un estudio del comportamiento dinámico del proceso sin que se pierda información útil del mismo. También se realizó un análisis en el dominio de las frecuencias, utilizando la transformada rápida de Fourier, el cual indicó que las mediciones realizadas no están bajo la influencia de ruidos. 

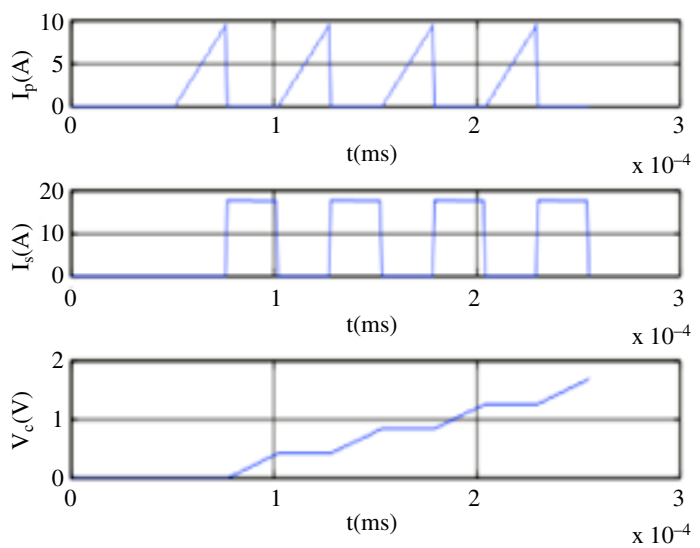

Figura 8. Parte de la evolución de las variables de estado.

El gráfico comparativo entre el promedio de las mediciones y la simulación del modelo se presenta en la Figura 10, donde se muestra la gran similitud que existe entre los valores de tensión obtenidos en la simulación del modelo y la curva promedio de las mediciones realizadas al proceso real.

Para reafirmar esto se realizó el cálculo de la función de correlación cruzada entre ambas curvas. Como se muestra en la Figura 11, existe una alta correlación entre el promedio de las mediciones y el resultado de la simulación. Esto afirma la similitud presente entre la salida del proceso y la del modelo.

Para determinar si con las desviaciones de la curva del promedio de las mediciones y el resultado de la simulación es posible utilizar el modelo obtenido como una representación del sistema, se calculó la media del error de simulación y el porciento que esta representa de la media de la salida real.

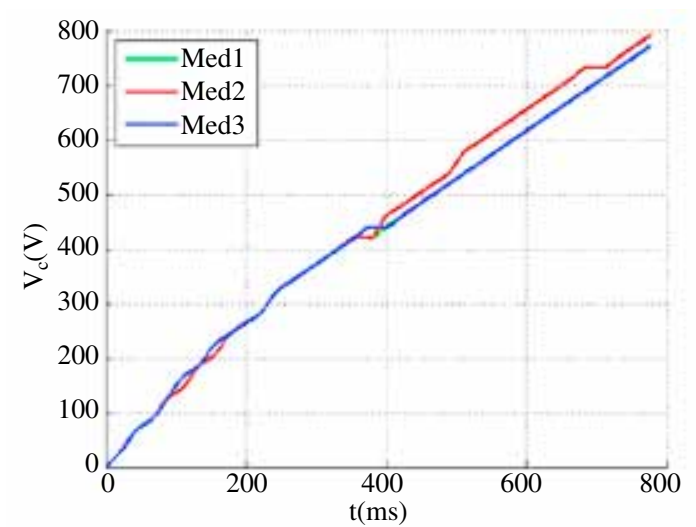

Figura 9. Gráfico de las mediciones del proceso.

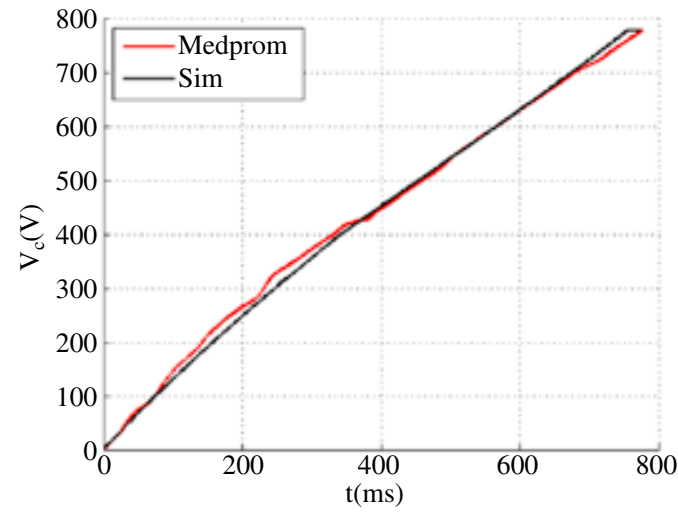

Figura 10. Gráfico comparativo entre el promedio de las salidas medidas y la simulada.

El valor medio del error de simulación es 5,88 V. Comparando este valor con el valor medio de la salida real, se pudo ver que este representa el 1,37\% del valor analizado de la salida real.

La Figura 12 muestra cómo se comportó el error de la simulación y el valor medio (Vm) del mismo.

Estos resultados demuestran que el modelo obtenido puede ser utilizado para representar el comportamiento del sistema real.

\section{Selección y diseño del diagnosticador}

Análisis de las diferentes alternativas de diagnóstico Los sistemas electrónicos, como lo es el sistema a diagnosticar, son extremadamente complejos para lograr un seguimiento de sus variables en tiempo real debido a que realizan sus operaciones en intervalos de tiempo muy pequeños, esto obliga a que las acciones de diagnóstico se realicen en el menor tiempo posible.

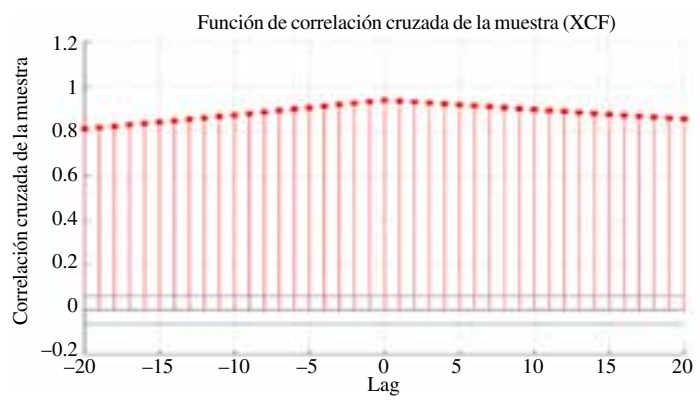

Figura 11. Función de correlación cruzada entre el promedio de las mediciones y la simulación. 


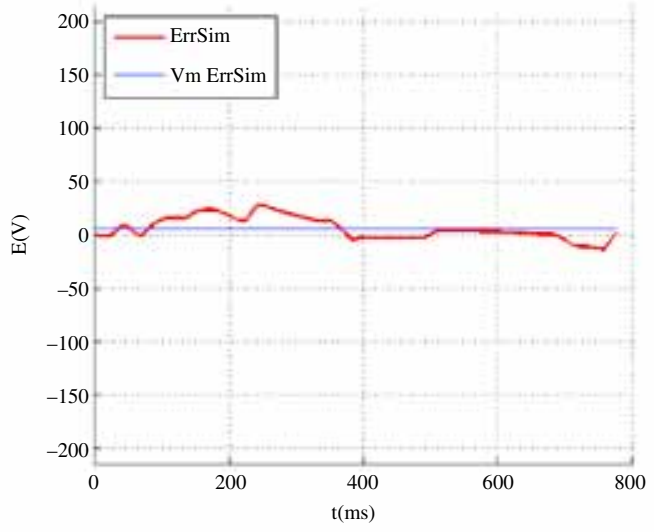

Figura 12. Error de simulación.

Existe una gama muy amplia de métodos utilizados en el diagnóstico de fallos que pueden clasificarse en tres grandes grupos:

- Métodos basados en modelos.

- Métodos basados en conocimiento.

- Métodos basados en datos históricos.

El diagnóstico de un sistema dinámico basado en modelo depende de forma crucial de la capacidad para estimar el estado del sistema dadas las observaciones.

La estimación para sistemas híbridos es particularmente compleja porque seguir el comportamiento de modelos múltiples y las transiciones entre los autómatas es computacionalmente muy caro. Simples extensiones de las técnicas de estimación convencionales, como el filtro de Kalman, conducen a algoritmos que requieren del seguimiento de todas las trayectorias posibles y, por tanto, son exponenciales en el número de pasos de tiempo. Además, se han utilizado aproximaciones gaussianas para estimar las distribuciones para cada trayectoria pero han tenido un rendimiento pobre. Debido a la demanda de gran capacidad de cómputo y que consumen mucho tiempo en su ejecución, características totalmente opuestas a las restricciones de operación que impone este equipo, los métodos enmarcados en los enfoques del primer y tercer grupo fueron descartados.

Dentro de los métodos que aparecen en el segundo grupo, compuesto en lo fundamental por técnicas de clasificación, podemos encontrar: los basados en el vecino y el prototipo más cercano [29], los basados en redes neuronales artificiales [30,31], los basados en reglas [32] y los clasificadores bayesianos [33]; y por técnicas de inferencia tales como: los árboles de fallos [34, 35] y los árboles de decisiones [36].

La estrategia seleccionada fue la de árboles de decisión. La selección se debe a que dentro de sus características está su fácil y rápida implementación porque se construyen a partir del conocimiento de los expertos, son estructuras que reflejan de manera intuitiva alguna decisión de clasificación y son fáciles de leer y entender.

Los árboles de decisión han sido ampliamente utilizados en el diagnóstico y se han empleado para sistemas electrónicos tal y como se aprecia en [36] donde se le añade al árbol de decisión información del comportamiento temporal del sistema. También se puede encontrar su aplicación combinado con otras técnicas como se presentan en [37, 38]. Para diseñar el diagnosticador del sistema analizado en este trabajo se utilizará la forma tradicional de implementación de los árboles de decisión ya que no es necesario utilizar versiones más sofisticadas de esta técnica.

\section{Diseño del diagnosticador}

El proceso de diseño del diagnosticador que se pretende utilizar consiste de dos pasos. En el primero, se genera fuera de línea una tabla de síntomas de fallo a partir del conocimiento de los expertos que fue corroborado con el análisis a partir del modelo como se explicó anteriormente. En el segundo paso, se compila un árbol de decisiones a partir de la tabla de síntomas de fallo, el cual es usado como diagnosticador en línea.

Paso 1: Generación de la tabla de síntomas de fallo. Un enfoque para diagnosticar sistemas es encontrar la hipótesis de fallo más probable a partir de las mediciones realizadas, las cuales pueden ser representadas de forma cualitativa. En el sistema bajo análisis las mediciones tienen la siguiente interpretación:

- Los eventos de fallos abruptos se representan por los valores binarios $(S)$ y $(N)$, donde $(S)$ indica la presencia de un fallo.

- Los fallos por degradación de los componentes se representan mediante el parámetro de fallo $\theta$, el cual tiene el siguiente significado:

(0) la variable que está siendo seguida se comporta de forma normal. 
(+) la variable que está siendo seguida tiene un valor por encima de lo normal.

(-) la variable que está siendo seguida tiene un valor por debajo de lo normal.

Las variables que están siendo seguidas $y_{k}$ son discretizadas y sus valores se representan a través de los valores cualitativos presentados anteriormente.

Autores como [39] son del criterio de que para diagnosticar algunos sistemas, es suficiente tener la interpretación cualitativa de los parámetros de fallo.

Los fallos de interés a detectar, así como los síntomas que lo indican se presentan en la Tabla 1.

Tabla 1. Tabla de síntomas de fallos para el bloque de carga del capacitor.

\begin{tabular}{|c|c|c|c|c|c|}
\hline $\begin{array}{c}\text { Id. } \\
\text { Fallo }\end{array}$ & $\begin{array}{c}\text { Componente } \\
\text { asociado }\end{array}$ & $\boldsymbol{y}_{\mathbf{1}}$ & $\boldsymbol{y}_{\mathbf{2}}$ & $\boldsymbol{y}_{\mathbf{3}}$ & $\boldsymbol{y}_{\mathbf{4}}$ \\
\hline F1 & Transf-Capac. & - & 0 & $\mathrm{~N}$ & $\mathrm{~N}$ \\
F1 & Transf-Capac. & 0 & 0 & $\mathrm{~N}$ & $\mathrm{~S}$ \\
\hline F2 & Oscilador & 0 & - & $\mathrm{N}$ & $\mathrm{N}$ \\
F2 & Oscilador & 0 & + & $\mathrm{N}$ & $\mathrm{N}$ \\
\hline F3 & Medidor & 0 & 0 & $\mathrm{~S}$ & $\mathrm{~N}$ \\
F3 & Medidor & + & 0 & $\mathrm{~N}$ & $\mathrm{~N}$ \\
F3 & Medidor & 0 & 0 & $\mathrm{~N}$ & $\mathrm{~S}$ \\
\hline
\end{tabular}

$y_{1}$ : Velocidad de carga del capacitor. Toma valores $\{-, 0,+\}$ para los casos: valor de tensión menor que el mínimo permitido, valor de tensión en el rango correspondiente al proceso de carga normal y valor de tensión mayor que el máximo posible, respectivamente.

$y_{2}$ : Duración de los niveles de los pulsos del oscilador. Toma valores $\{-, 0,+\}$ para los casos: la salida del oscilador se mantuvo en nivel bajo un tiempo mayor que el permitido $\left(t_{p}>T\right)$, el tiempo de duración de la salida del oscilador en ambos niveles corresponde con el funcionamiento normal del mismo y la salida del oscilador se mantuvo en nivel alto un tiempo mayor que el permitido (ton $>0.75 T$ ), respectivamente. Todo esto es determinado a través de la medición de la corriente en el devanado primario del transformador $\left(i_{p}\right)$.

$y_{3}$ : Tensión máxima permitida. Toma el valor $S$ si la tensión en el capacitor sobrepasa la carga deseada y llegó a su valor máximo permitido y $N$ de lo contrario.

$y_{4}$ : Tiempo máximo de carga permitido. Toma el valor $S$ si el proceso de carga se demora más que el tiempo máximo permitido $\left(t_{c}>5 s\right)$ y $N$ de lo contrario.

Como se puede apreciar en la tabla de síntomas de fallos, existen dos fallos diferentes que, con la información que se dispone a partir de la disposición de los sensores, presentan los mismos síntomas. Esto significa que el sistema bajo análisis no es totalmente diagnosticable debido a que existen dos fallos diferentes que son detectables pero que no hay información suficiente que permita diferenciar cuál de los dos es el que realmente ha ocurrido.

Lo anterior indica que el uso de este enfoque permite desde la etapa de diseño saber cuáles son las potencialidades reales de diagnóstico que posee el sistema bajo las restricciones actuales impuestas al mismo y permite al equipo diseñador tomar las medidas pertinentes. En el caso que nos ocupa después de evaluar desde un punto de vista costobeneficio lo que significaría agregar un sensor más para poder diferenciar cuál de los fallos ocurrió se decidió no agregar el mismo.

Paso 2: Construcción del árbol de decisión.

Para el diseño de estructuras de árboles se han propuesto muchos métodos. En este caso se implementó un algoritmo del tipo "top-down" para la generación del árbol de decisión que se utilizó como diagnosticador para el sistema. En este tipo de método el diseño del árbol de decisión se reduce a las siguientes tareas:

1. Selección de un nodo.

2. Decisión de cuáles nodos son terminales.

3. Asignación a cada nodo terminal de un identificador de clase.

Para seleccionar la prueba que ofrece mayor información se usó el algoritmo ID3 [40]. Este algoritmo examina todas las pruebas candidatas y escoge la prueba que tiene la menor incertidumbre en la decisión y con esto se logra minimizar la profundidad del árbol, lográndose un árbol de decisión que realiza la menor cantidad de encuestas a los sensores y que ofrece la información más rápidamente.

Aplicando los algoritmos mencionados anteriormente se obtuvo el árbol de decisión que se muestra en la Figura 13, donde el valor $N$ como salida del sensor $y_{4}$ indica el estado de operación normal del sistema. 


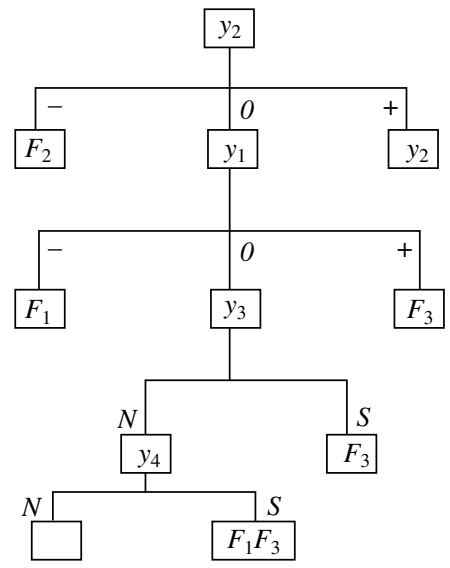

Figura 13. Diagnosticador basado en árboles de decisión para el bloque de carga del capacitor.

\section{Evaluación del desempeño del diagnosticador}

Para realizar una evaluación del desempeño del diagnosticador se realiza una simulación del modelo del sistema aumentándolo con un diagnosticador construido a partir del árbol obtenido. Para esto, se divide el estado representativo de la ocurrencia de un fallo, $q_{F}$ en la Figura 7, en los diferentes estados de fallos a detectar. El árbol de decisiones obtenido indica los valores de lectura de los sensores que implican fallos, esta información es utilizada para establecer la transición hacia el estado de fallo correspondiente.

Esto permitirá inducir diferentes condiciones de fallo y verificar que utilizando la lectura de los sensores previstos se obtienen los resultados de diagnóstico esperados.

El autómata ampliado con el diagnosticador se muestra en la Figura 14. En este esquema los estados $q_{F_{i}}$ representan los modos de fallos a detectar basándose en el patrón presente en las mediciones. Las transiciones hacia cada uno de estos estados se habilitan en dependencia del valor los sensores. El árbol de decisiones obtenido indica hacia qué estado debe realizarse la transición cuando las mediciones presentan valores fuera de los rangos establecidos como operación normal. Por ejemplo, si en el estado $q_{1}$ el sensor $y_{1}$ registra lectura que significa un comportamiento no adecuado en la velocidad de carga del capacitor, entonces se puede transitar, según muestra el árbol de decisión a los estados $q_{F_{1}}$ o $q_{F_{3}}$.

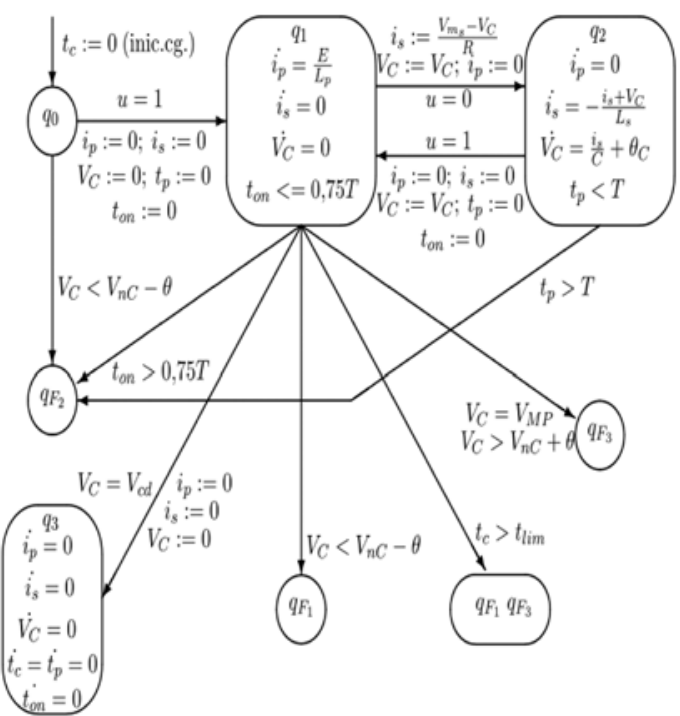

Figura 14. Modelo del sistema aumentado con el diagnosticador.

Para validar el funcionamiento del diagnosticador se definió el autómata finito mostrado en la Figura 15, el cual está compuesto por el conjunto de estados discretos

$Q=\left\{q_{0}, q_{1}, q_{2}, q_{3}, q_{F_{1}}, q_{F_{2}}, q_{F_{3}}, q_{F_{1} F_{3}}\right\}$

los cuales representan tanto el funcionamiento normal como defectuoso del sistema, haciendo abstracción del comportamiento dinámico del mismo.

Para el autómata se define el conjunto de eventos $E=\{a, b, c, d, e, f, g, h, i\}$

cuyos significados se describen a continuación:

$$
\begin{aligned}
& a \Rightarrow u=1 \\
& b \Rightarrow u=0 \\
& c \Rightarrow V_{C}=V_{c d} \\
& d \Rightarrow V_{C}<V_{n c}-\theta \\
& e \Rightarrow V_{C}>V_{n c}+\theta \\
& f \Rightarrow V_{C}=V_{M P} \\
& g \Rightarrow t_{o n}>0.75 T \\
& h \Rightarrow t_{p}>T \\
& i \Rightarrow t_{c}>t_{\lim }
\end{aligned}
$$


Para el autómata se definen las transiciones

$$
\begin{array}{lll}
\delta\left(q_{0}, a\right)=q_{1} & \delta\left(q_{0}, d\right)=q_{F_{2}} & \delta\left(q_{1}, b\right)=q_{2} \\
\delta\left(q_{1}, c\right)=q_{3} & \delta\left(q_{1}, g\right)=q_{F_{2}} & \delta\left(q_{1}, d\right)=q_{F_{1}} \\
\delta\left(q_{1}, e\right)=q_{F_{3}} & \delta\left(q_{1}, f\right)=q_{F_{3}} & \delta\left(q_{1}, i\right)=q_{F_{1} F_{3}} \\
\delta\left(q_{2}, a\right)=q_{1} & \delta\left(q_{2}, h\right)=q_{F_{2}} &
\end{array}
$$

Siendo el conjunto de estados finales del autómata

$$
F=\left\{q_{3}, q_{F_{1}}, q_{F_{2}}, q_{F_{3}}, q_{F_{1} F_{3}}\right\}
$$

el cual está compuesto por el estado que indica la carga exitosa del capacitor y los diferentes estados de fallos.

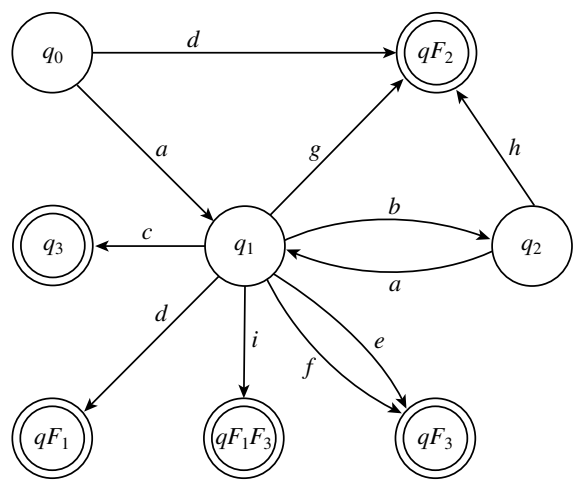

Figura 15.Esquema del autómata finito del diagnosticador.

El funcionamiento del diagnosticador se comprobó utilizando el verificador spin, el cual fue desarrollado en los laboratorios Bell, en Estados Unidos por Gerard J. Holzmann [41], en la cual se implementó el modelo del autómata y se comprobó el comportamiento del diagnosticador para las posibles secuencias de eventos que pudieran presentarse en el sistema. En este trabajo se presentan, a manera de ejemplo, dos de las posibles secuencias de eventos.

La primera secuencia representa un proceso de carga exitosa, el cual está definido por la secuencia $a \rightarrow b \rightarrow a \rightarrow b \rightarrow a \rightarrow c$. Para dicha secuencia la herramienta arrojó el siguiente resultado

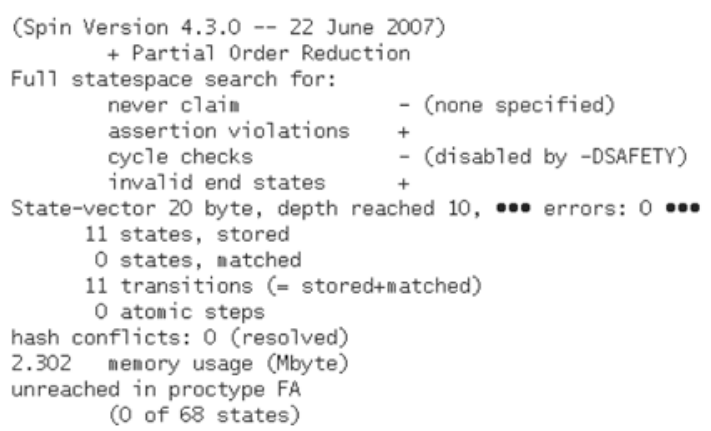

La segunda secuencia representa un proceso de carga donde se realiza una lectura incoherente del valor de la tensión en el capacitor, el cual está definido por la secuencia $a \rightarrow b \rightarrow a \rightarrow b \rightarrow a \rightarrow e$. Para dicha secuencia la herramienta arrojó el siguiente resultado

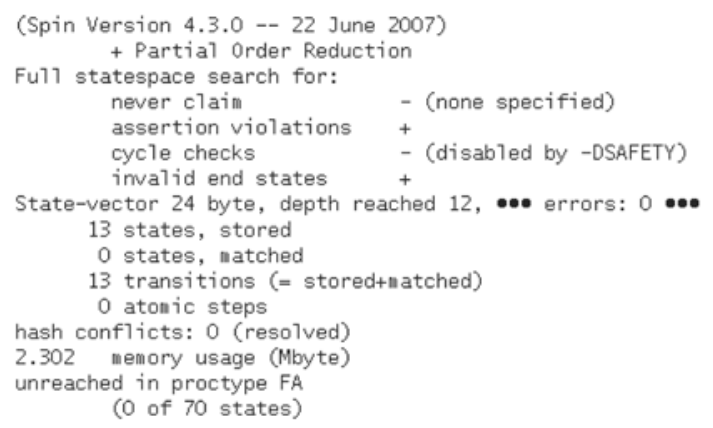

Como se puede apreciar ningún error fue encontrado durante la búsqueda efectuada a través de los estados que se alcanzaron del modelo durante las simulaciones y se alcanzó el estado final deseado. Esto demuestra el éxito del diagnóstico en encontrar los fallos inducidos y la viabilidad de la implementación del diagnosticador propuesto.

La secuencia de encuestas a los sensores, indicada por el árbol de decisión obtenido, se encuentra implementada en el programa de control de la carga en el equipo desfibrilador, mostrando resultados satisfactorios en su desempeño.

Posteriormente se tomó el autómata representado por el modelo del sistema con sus dinámicas asociadas aumentado con el diagnosticador (Figura 14) y se cambiaron los parámetros que representan las secuencias de eventos anteriores comprobándose en el modelo híbrido del sistema con el diagnosticador incorporado el funcionamiento correcto de este 
último. Todo lo anterior se simuló utilizando el paquete Stateflow Toolbox de MatLab 7.0. [28]

\section{CONCLUSIONES}

La contribución fundamental de este trabajo es presentar un enfoque para el diseño de un equipo de desfibrilación ventricular donde se incorpora el concepto de diseño basado en criterios de diagnóstico de fallos y se utilizan los autómatas híbridos y su composición para obtener el modelo del sistema.

La incorporación del concepto de diseño basado en criterios de diagnóstico de fallos posibilita fijar las pautas necesarias desde la fase de diseño que permiten implementar un sistema de diagnóstico de fallos y que representa una ventaja adicional con respecto al sistema tradicional de protecciones que tienen muchos sistemas electrónicos de alta complejidad para los temas de mantenimiento y reparación.

Dentro del procedimiento propuesto uno de los pasos es realizar el modelado del sistema. En este trabajo se combinó el conocimiento de los expertos que permitió definir los bloques funcionales del sistema analizado y el modelado mediante la composición de autómatas híbridos que permite reducir el grado de complejidad en la representación del comportamiento dinámico del sistema que se está modelando. El autómata paralelo resultante permitió realizar simulaciones para estudiar fuera de línea los efectos de los posibles fallos que se debían producir en el sistema que se estaba diseñando. También se validó el modelo comprobándose que los niveles de error en el modelado estaban dentro de un rango aceptable. Los satisfactorios resultados que se obtuvieron avalan la elección de las herramientas seleccionadas.

En la fase de diseño del diagnosticador es necesario analizar las características del sistema a diagnosticar para la selección del método a utilizar. En el caso presentado, se argumenta que no es posible realizar un diagnóstico basado en modelo o datos históricos por la demanda de potencia de cómputo y consumo de tiempo que estos enfoques requieren. Por lo que la estrategia de diagnóstico seleccionada basa su funcionamiento en un árbol de decisión. El árbol fue construido a partir de la tabla de síntomas de fallos, la cual captura las condiciones actuales de diseño y refleja las posibilidades de diagnóstico, utilizando como método de selección de las pruebas el algoritmo ID3.

Esta estrategia permite utilizar las protecciones tradicionalmente implantadas en los equipos para, de conjunto con el conocimiento experto, proporcionar una interpretación de las violaciones de las mismas. Con lo anterior se logra ofrecer un diagnosticador de fallos en correspondencia con las potencialidades de cómputo que posee el equipo, lo cual es cualitativamente superior a un sistema de protecciones y por lo tanto resulta en un equipo con mejores prestaciones.

Finalmente es necesario destacar que la aplicación del procedimiento propuesto evita los problemas que fueron enunciados en la introducción del trabajo y que el mismo puede ser extendido para su aplicación a otros sistemas que no sean necesariamente sistemas electrónicos.

\section{AGRADECIMIENTOS}

Los autores agradecen al Instituto Central de Investigación Digital por su apoyo en el desarrollo de este trabajo.

\section{REFERENCIAS}

[1] R. Isermann. "Process fault detection based on modeling and estimation methods -a survey". Automatica. Vol. 20, Issue 4, pp. 387-404. 1984. ISSN: 0005-1098.

[2] R. Isermann and P. Ballé. "Trends in the application of model-based fault detection and diagnosis of technical processes". Control Eng. Practice. Vol. 5, pp. 709-719. 1997. ISSN: 0967-0661.

[3] J. Chen and R.J. Patton. "Robust Model-Based Fault Diagnosis for Dynamic Systems". Kluwer Academic Publisher. 1999.

[4] J. Gertler. "Fault detection and isolation using parity relations". Control Eng. Practice. Vol. 5, pp. 653-661. 1997. ISSN: 0967-0661.

[5] R.J. Patton and J. Chen. "Observer based fault detection and isolation -robustness and applications". Computers and Chemical Engineering. Vol. 5, pp. 671-682. 1997. ISSN: 0098-1354.

[6] S. Simani, C. Fantuzzi and R.J. Patton. "Model-Based Fault Diagnosis in Dynamic 
Systems Using Identification Techniques". Springer-Verlag. London, UK. 2002.

[7] V. Venkatasubramanian, R. Rengaswamy, K. Yin and S.N. Kavuri. "A review of process fault detection and diagnosis - Part I quantitative model-based methods". Computers and Chemical Engineering. Vol. 27, pp. 293-311. 2003. ISSN: 0098-1354.

[8] V. Venkatasubramanian, R. Rengaswamy and S.N. Kavuri. "A review of process fault detection and diagnosis - Part II qualitative models and search strategies". Computers and Chemical Engineering. Vol. 27, pp. 313326. 2003. ISSN: 0098-1354.

[9] V. Venkatasubramanian, R. Rengaswamy, S.N. Kavuri and K. Yin. "A review of process fault detection and diagnosis - Part III process history based methods". Computers and Chemical Engineering. Vol. 27, pp. 327-346. 2003. ISSN: 0098-1354.

[10] J. Chen and C.M. Liao. "Dynamic process fault monitoring based on neural network and PCA". Journal of Process Control. Vol. 12, pp. 277-289. 2002. ISSN: 0959-1524.

[11] A. Alessandri. "Fault diagnosis for nonlinear systems using a bank of neural estimators". Computers in Industry. Vol. 52, pp. 271-289. 2003. ISSN: 0166-3615.

[12] A. Skoglund, A. Brundin and C.F. Mandenius. "Applying process monitoring with multivariate analysis through a knowledgebased systems approach to a paperboard machine". Computers in Industry. Vol. 56, pp. 472-478. 2005. ISSN: 0166-3615.

[13] B.H. Krogh. "Recent advances in discrete analysis and control of hybrid systems". IEEE Sixth International Workshop on Discrete Event Systems (WODES'02), pp. 311-314. Zaragoza, Spain. October 2-4, 2002.

[14] T.A. Henzinger. "The theory of hybrid automata". In: Proc. 11th Annual Symp. Logic Computer Science. IEEE Control Systems Society, pp. 278-292. New Brunswick, New Jersey, USA. July 27-30, 1996.

[15] R. Alur. "Timed automata". 11th International Conference on Computer Aided Verification, LNCS 1633, pp. 8-22. Springer-Verlag. 1999.

[16] B.R. Bakshi. "Multiscale PCA with application to multivariate statistical process monitoring". AIChE Journal. Vol. 44, pp. 1596-1610. 1998. ISSN: 0001-1541.
[17] J. Chen and K.C. Liu. "On-line batch process monitoring using dynamic PCA and dynamic PLS models". Chemical Engineering Science Vol. 57, pp. 63-75. 2002. ISSN: 0009-2509.

[18] W.R. Becraft and P.L. Lee. "An integrated neural network-expert system approach for fault diagnosis". Computers and Chemical Engineering. Vol. 17, pp. 1001-1014. 1993. ISSN: 0098-1354.

[19] C. Gertosio and A. Dussauchoy. "Knowledge discovery from industrial databases". Journal of Intelligent Manufacturing. Vol. 15, pp. 29-37. 2004. ISSN: 0956-5515.

[20] J. Folgueras. "CARDIODEF 2 desfibriladormonitor bifásico". Reporte de Investigación Instituto Central de Investigación Digital. 2004.

[21] J. Folgueras, A.E. Portela, O. Colorado, S. Fornells, A. Ruiz y A. Milanés. "Sistema y método para aparato de electroterapia". Oficina Cubana de la Propiedad Industrial, No. 22981. La Habana, Cuba. 2004.

[22] J.M. Owen, R.W. Fincke, J.P.O. Leary and M.H. Totman. "Desfibrillation system". Patent Application Publication. Pub. No.: US 2003/0004547 A1. 2003.

[23] I. Hwang, H. Balakrishnan and C. Tomlin. "State estimation for hybrid systems: applications to aircraft tracking". IEE Proc. - Control Theory and Applications. Vol. 153, No. 5, pp. 556-566. 2006. ISSN: 1350-2379.

[24] A. Balluchi, L. Benvenuti, M.D. Benedetto, C. Pinello, A. Luigi and S. Vincentelli. "Automotive engine control and hybrid systems: Challenges and opportunities". In Proceedings of the IEEE. Vol. 88, pp. 888912. 2000.

[25] J. Lygeros, D.N. Godbole and S. Sastry. "Verified hybrid controllers for automated vehicles". IEEE Transactions on Automatic Control. Vol. 43, pp. 522-539. 1998. ISSN:0018-9286.

[26] T. Bak, J.D. Bendtsen and A.P. Ravn. "Hybrid control design for a wheeled mobile robot". Hybrid Systems: Computation and Control (HSCC 2003). LNCS 2623, pp. 50-65. Springer-Verlag. 2003. ISSN: 1861-2059.

[27] N. Mohan, T.M. Undeland and W.P. Robbins. "Power electronics converters, applications and design". Second Ed. John Wiley and Sons Inc. 1995. 
[28] The Math Works, Inc. "Stateflow For Use with Simulink Toolbox User's Guide”. 6 Ed. 2005.

[29] J.M. House, W.Y. Lee and D.R. Shin. "Classification techniques for fault detection and diagnosis of an air-handling unit". ASHRAE Transactions. Vol. 105, pp. 19871997. 1999. ISSN: 0001-2505.

[30] T. Sorsa and H.N. Koivo. "Application of artificial neural networks in process fault diagnosis". Automatica. Vol. 29, pp. 843849. 1993. ISSN: 0005-1098.

[31] W.Y. Lee, J.M. House and D.R. Shin. "Fault diagnosis and temperature sensor recovery for an air-handling unit". ASHRAE Transactions. Vol. 103, pp. 621-633. 1997. ISSN: 0001-2505.

[32] W.C. Chen, S.S. Tseng and C.Y Wang. "A novel manufacturing defect detection method using association rule mining techniques". Expert Systems with Applications. Vol. 29, pp. 807-815. 2005. ISSN: 0957-4174.

[33] E. Charniak. "Bayesian networks without tears". AI Magazine. Vol. 12, pp. 50-63. 1991. ISSN 0738-4602.

[34] R. de Vries. "An automated methodology for generating a fault tree". IEEE Trans. Reliability. Vol. 39, pp. 76-86. 1990. ISSN: 0018-9529.

[35] R. Isermann. "Model-based fault detection and diagnosis - status and applications". Annual
Reviews in Control. Vol. 29, pp. 71-85. 2005. ISSN: 1367-5788.

[36] L. Console, C. Picardi and D.T. Dupré. Temporal decision trees: Model-based diagnosis of dynamic systems on board". Journal of Artificial Intelligence Research. Vol. 19, pp. 469-512. 2003. ISSN: 1076-9757.

[37] B.S. Yang, D.S. Lim and A.Ch. Chiow. "VIBEX: an expert system for vibration fault diagnosis of rotating machinery using decision tree and decision table". Expert Systems with Applications. Vol. 28, pp. 735742. 2005.

[38] Y. Zhou, J. Hahn and M.S. Mannan. "Process monitoring based on classification tree and discriminant analysis". Reliability Engineering and System Safety. Vol. 91, pp. 546-555. 2006.

[39] X. Koutsoukos, F. Zhao, H. Haussecker, J. Reich and P. Cheung. "Fault modeling for monitoring and diagnosis of sensor-rich hybrid systems". In Conference on Decision and Control (40th IEEE, Ed.). 2001.

[40] J.R Quinlan. "Induction of decision trees". Machine Learning. Vol. 1, pp. 81-106. 1986. ISSN: 0885-6125.

[41] G.J. Holzmann. "The model checker spin". IEEE transaction on software engineering. Vol. 5, pp. 11-20. 2006. 